\title{
Face and Eye Recognition on Gray Image using DWT with RBFSVM Method
}

\author{
Naveen Kumar Ahirwar ${ }^{1}$ and Manish Dixit ${ }^{2}$ \\ Department of Computer Science Engineering and Information Technology \\ Madhav Institute of Technology \& Science, Gwalior, India \\ 'naveenkumarahirwar99@gmail.com, ${ }^{2}$ dixitmits@gmail.com
}

\begin{abstract}
Facial part detection or extraction shows the most important role in face and eye recognition. In this article proposed a new algorithm for Face and Eye Recognition (FER) using radial basis function support vector machine (RBFSVM) classifier. The discrete wavelet transform (DWT) is used for feature extraction and selection. For the experimental results, used JAFFE and ORL database. In this algorithm, extract face component like left eye, right eye, mouth and nose. In the preprocessing stage, apply median filtering for removing noise from an image. This stage improves the feature extraction process. Finding an image from the image components is a typical task in pattern recognition. The detection rate has reached up tol00\% for eye recognition and for face recognition is $90-96 \%$. The proposed system estimates the value of precision and recall. This algorithm is compared with SVM and our proposed proved better than previous algorithms.
\end{abstract}

Keywords: Median Filter, RBFSVM, DWT (Discrete Wavelet Transform), Precision, Recall

\section{Introduction}

In biometrics systems, machine learning techniques can be effectively used in feature extraction and classification. Biometrics deals with the recognition of people based on physiological and behavioral characteristics. Biometric recognition uses automated methods for recognition and this is why it is much related to machine learning [1]. Face and Eye recognition system is a pattern recognition technique which is developed based on some mathematical model and has two vital steps i.e. feature extraction and classification. The image processing methods has been applied to extract features from face database and some classification techniques are applied to recognize the unknown facial template. In recent year, various different research studies have been performed to develop the FER system. The dimension of the face image is the vital issue to resist the performance of the recognition system. PCA has been used to reduce the dimension of the face database and extracted the feature face that is used in FER technique [2].

SVM have been recently proposed by Vapnik and his co-workers [4] as efficient technique for all pattern recognition. The work is focused on problem of eye recognition, and show that the discrimination functions learned by RBFSVM can give much higher recognition accuracy than the popular standardeigen-face approach using kernel SVM classifier. PCA is used to extract the feature from the face image. After the features are extracted, the RBFSVM are learned and the disjoint test set enters the system for recognition. We propose to construct a one versus all to recognize the testing samples. We perform experiments on the JAFFE and ORL face database [3]. 


\section{Related Work}

Shuo Chen (2014)-This paper first proposes three clustering-based discriminatory analysis (CDA) models to address the problem of inadequate features derived from the FLD. The CDA-1 model defines a new within-cluster scatter matrix Scwand a new between-cluster scatter matrix $S c b$ by means of the $k$-means clustering technique. The rank of the Scbincreases as the number of clusters increases, and therefore the CDA-1 can derive adequate features for achieving satisfactory performance [4].

N K Bansode (2014)-In this paper, it proposed a new approach for face feature description using SVM classifier [5].The left eye, right eye, left eyebrow, the right eyebrow detection rate is less due to occlusion of the eyes and eyebrows due to hair.

I. PauknerStojkov (2010)-This paper proposes a two-step solution. The first step is applying Bayesian classifiers and connected components labeling for determining the face position [6]. In detection process all of the components of the selected color space are used.The detection rate is too less for eye.

KhairulAzha A. Aziz (2009)-This paper presents a face detection system using RBFNN with variance spreadvalue [7]. Face detection is the first step in face recognition system. The face region is localized and extract from the background that will be fed into the face recognition system for identification.The system may give better results if the overlapping factors are taken into consideration for future research [8].

\section{Proposed Methodology}

In the proposed system, there are four stages for performing operations on image:,

Preprocessing

Feature Extraction

Feature Selection

Classification

\section{Preprocessing}

In this stage, first take an image and is to be filtered by median filtering. After that resize the image in the standard size is $200 * 200$. This stage is repeated for train images also.

\section{Feature Extraction}

In this stage, filtered image is used for feature extraction process. Extract the features of an image using DWT method. DWT decompose the image into four bands: low pass filter and high pass filter. It is a lossless compression method; this method does not degrade the quality of an image.

\section{Feature Selection}

This stage selects feature of an image using Manhattan distance. This distance is used for selecting test image feature and database image feature and calculate distance between test image and database image.

\section{Classification}

In this process, classify data using RBFSVM. It accomplishes pattern recognition between two classes by finding a decision surface that has maximum distance to the closest points in the training set which are termed support vectors. The block diagram of proposed method is shown in Figure 1. 


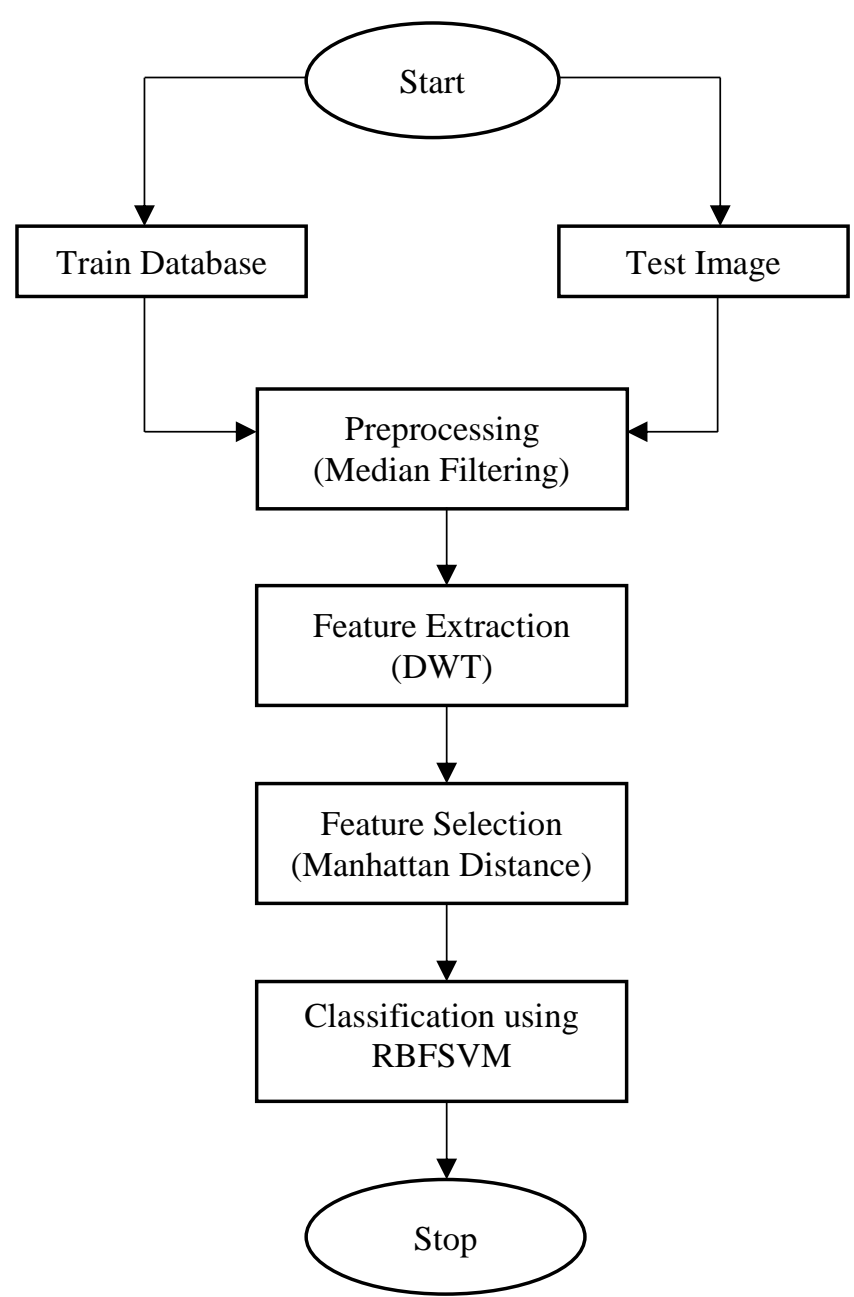

Figure 1. The Block Diagram of our Proposed Method

\section{Proposed Algorithm}

1. Read test image from train database.

2. Apply median filter on test image and resize the test image.

3. Extract feature of test image using discrete wavelet transform which is divide image into four sub bands: [Coeff1, Coeff2, Coeff3, Coeff4]

4. Take train database images which contain 60 images of 10 persons and test database which contain crop images of 10 persons.

5. In the preprocessing step, apply a median filter for removing noise from an image on train database.

6. Resize all train images with size $200 * 200$.

7. In the feature extraction process, extract features of an image using DWT with mean and standard deviation.

8. Repeat step 5 to 7 until all train image processing is completed.

9. In feature selection, calculate Manhattan distance between test image and train images using below equation: 
$\operatorname{Manhattan}(k)=\frac{\operatorname{sum}(\operatorname{abs}(x(k,:)-s))}{(1+x(k,:)+s))}$

Where $\mathrm{k}$ varies from 1 to number of images, $\mathrm{x}$ is feature of train database and $\mathrm{s}$ is test feature.

10. In classification process, apply SVM with RBF for classifying the data.

11. Calculate the average precision and recall of the system using below formula:

$P=\frac{\text { No. ofrelevantimageretrieved }}{\text { Totalnumberofimageretrieved }}$

$R=\frac{\text { No. ofrelevantimageretrieved }}{\text { numberofimageinthedatebese }}$

12. Calculate detection rate (DR) of the system using this formula:

$D R=100 \times\left(\frac{T P+T N}{N}\right)$

Where TP is true positive and TN is true negative and $\mathrm{N}$ is size of dataset.

\section{Image Dataset}

The Standard Image dataset is taken to perform evaluation of our proposed methodology.

Image Dataset of Train images

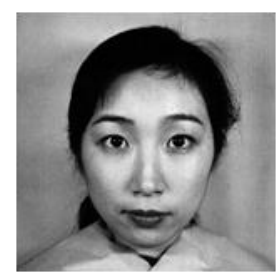

1.tiff

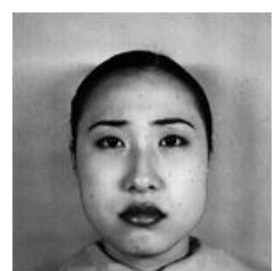

10.tiff

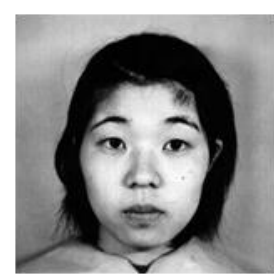

3.tiff

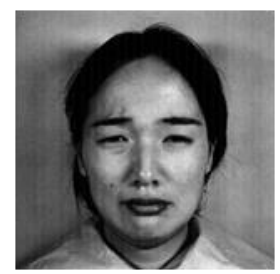

11.tiff

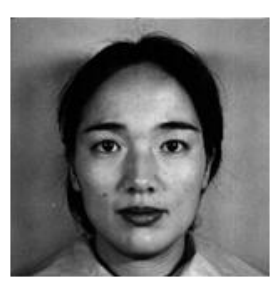

5.tiff

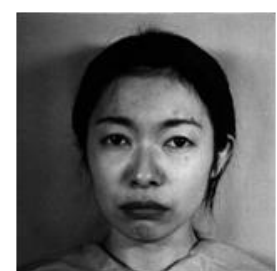

12.tiff

Figure 2. JAFFE Image Dataset 
Preprocessing Step for Test Images

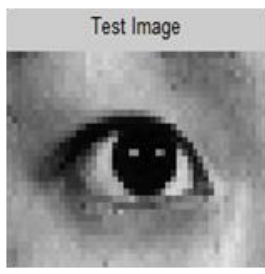

(a)

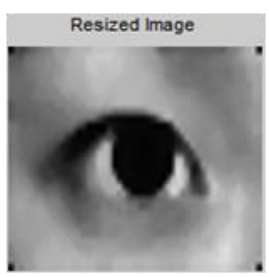

(c)

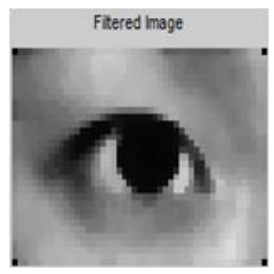

(b)

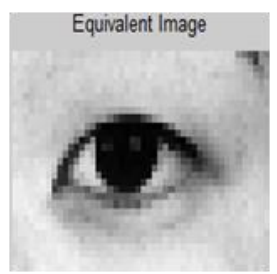

(d)

Figure 3. (a) Input Image (b) Filtered Image (c) Resized Image(d) Equivalent Image

Preprocessing Step for Test Images

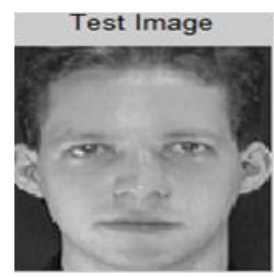

(a)

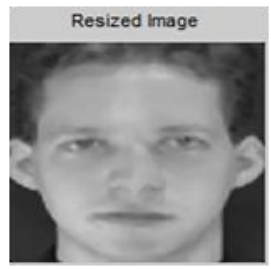

(c)

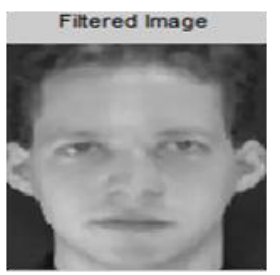

(b)

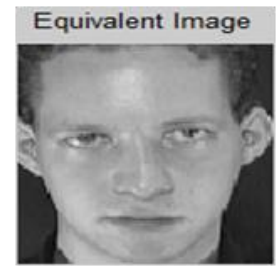

(d)

Figure 4. (a) Input Image (b) Filtered Image (c) Resized Image(d) Equivalent Image 
Table 1. Face and Face Features

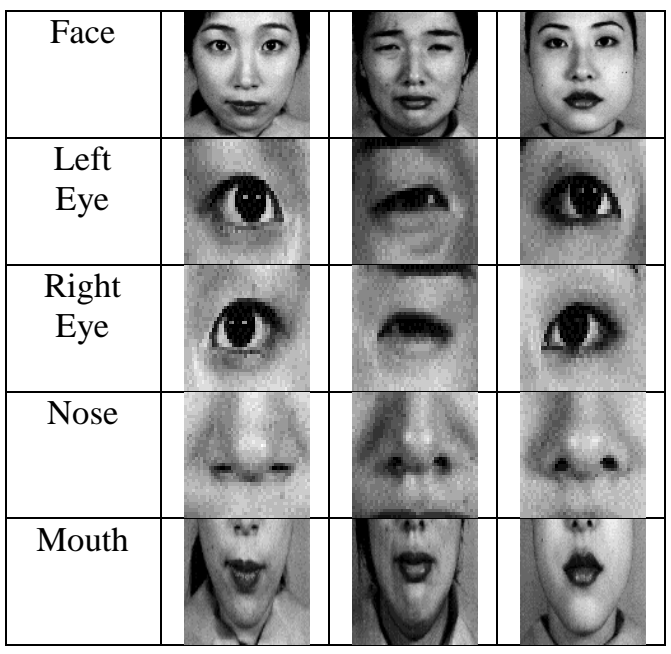

\section{Performance Evaluation}

MATLAB is a data visualization and analysis tool which has been designed with powerful support for matrix and matrices operations. Along with this, Matlab has capabilities of excellent graphics, and its own powerful programming language. One of the reasons that Matlab has become such an important tool is by the use of Matlab programs designed sets to support a specific task. These programs sets are known as toolboxes, and the specific interest toolbox to us is the image processing toolbox.

\section{Table 2. Detection Rate Comparison between Base and Proposed System}

\begin{tabular}{|c|c|c|}
\hline Train Image & $\begin{array}{c}\text { Base } \\
\text { Detection Rate } \\
\text { (in \%) }\end{array}$ & $\begin{array}{c}\text { Proposed } \\
\text { Detection Rate } \\
\text { (in \%) }\end{array}$ \\
\hline 10.tiff & 36.67 & 90.00 \\
\hline 11.tiff & 46.67 & 93.33 \\
\hline 21.tiff & 36.67 & 86.67 \\
\hline 28.tiff & 26.67 & 90 \\
\hline 36.tiff & 43.33 & 96.67 \\
\hline 43.tiff & 26.67 & 93.33 \\
\hline 8.tiff & 26.67 & 93.33 \\
\hline 50.tiff & 46.67 & 96.67 \\
\hline 31.tiff & 30 & 96.67 \\
\hline 29.tiff & 33.33 & 100 \\
\hline
\end{tabular}

Table 3. Precision and Recall Comparison between Base and Proposed System

\begin{tabular}{|c|c|c|c|c|}
\hline $\begin{array}{c}\text { Train } \\
\text { Image }\end{array}$ & $\begin{array}{c}\text { Base } \\
\text { Precision } \\
\text { (in \%) }\end{array}$ & $\begin{array}{c}\text { Proposed } \\
\text { Precision } \\
\text { (in \%) }\end{array}$ & $\begin{array}{c}\text { Base } \\
\text { Recall } \\
\text { (in \%) }\end{array}$ & $\begin{array}{c}\text { Proposed } \\
\text { Recall } \\
\text { (in \%) }\end{array}$ \\
\hline 10.tiff & 0 & 100 & 0 & 66.66 \\
\hline 11.tiff & 40 & 75 & 33.33 & 100 \\
\hline 21.tiff & 40 & 100 & 33.33 & 100 \\
\hline 28.tiff & 0 & 75 & 0 & 100 \\
\hline 36.tiff & 33.33 & 100 & 50 & 100 \\
\hline 43.tiff & 33.33 & 100 & 16.66 & 100 \\
\hline
\end{tabular}




\begin{tabular}{|c|c|c|c|c|}
\hline 8.tiff & 38.46 & 100 & 83.33 & 83.33 \\
\hline 50.tiff & 42.85 & 100 & 50 & 100 \\
\hline 31.tiff & 16.66 & 100 & 16.66 & 100 \\
\hline 29.tiff & 33.33 & 100 & 50 & 100 \\
\hline
\end{tabular}

Table 4. Proposed Results on Precision, Recall and Detection Rate

\begin{tabular}{|c|c|c|c|}
\hline $\begin{array}{c}\text { ORL } \\
\text { Image }\end{array}$ & $\begin{array}{c}\text { Precision } \\
\text { Rate (in } \\
\% \text { ) }\end{array}$ & $\begin{array}{c}\text { Recall } \\
\text { Rate (in } \\
\% \text { ) }\end{array}$ & $\begin{array}{c}\text { Detection } \\
\text { Rate (in } \\
\%)\end{array}$ \\
\hline 83.33 & 50 & 60 \\
\hline & 83.33 & 50 & 60 \\
\hline & & & \\
\hline & & & \\
\hline & & & \\
\hline
\end{tabular}

The result obtained of precision and recall value using proposed methodology is shown in table 4 . The figure 5 shows bar graph of detection rate obtain using proposed, method compare with base method. 


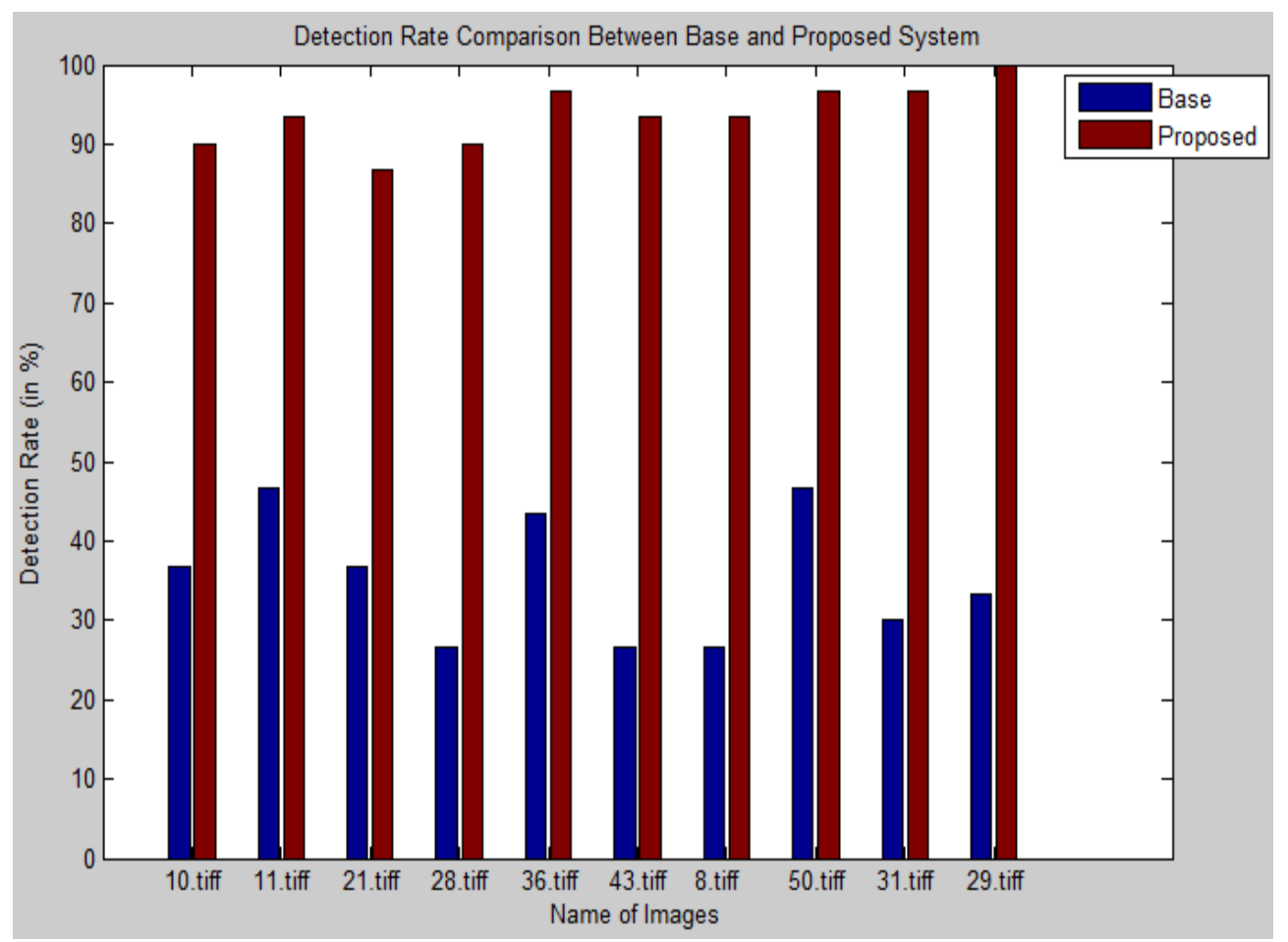

Figure 5. The Bar Graph Shows Detection Rate Comparison between Base and Proposed System

It shows that the detection rates of RBFSVM are much higher than that of Kernel SVM (KSVM). If we choose the best results among the results, the highest classification rate of the RBFSVM can reach $100 \%$ whereas KSVM gives $99 \%$.

\section{Conclusion}

In this study, we have proposed method for face and eye recognition We have used face and eye detection algorithm and feature extraction methods for the analysis of the features. In this algorithm, using RBFSVM classifier and DWT with Manhattan is used for feature extraction and selection.The RBFSVM classifier for a textual description of facial feature attributes such as left eye, right eye, mouth, face and nose based on the normalized face width value. This description of the facial feature can be used to find and retrieve related face image using high level concepts in the context search.

In this proposed system, worked on two databases: JAFFE and ORL. In the first database, take 10 person images with face parts. In second database, take 20 images which is labeled by Not Matched while 10 images which is labeled by Matched. Furthermore, we will work on ORL database with different feature algorithm and classifier. 


\section{References}

[1] M. Oravec, "Feature Extraction and Classification by Machine Learning Methods for Biometric Recognition of Face and Iris", 56th International Symposium ELMAR-2014, Zadar, Croatia, (2014) September, pp. 1-4.

[2] R. Toufiq and Md. R. Islam, "Face Recognition System using PCA-ANN Technique with Feature Fusion Method", ICEEICT (2014), pp. 1-5.

[3] Md. O. Faruqe, Md. A. M. Hasan, "Face Recognition Using PCA and SVM", ,DOI-10.1109/ICASID, (2009), pp. 97-101.

[4] S. Chen and C. Liu, "Clustering-Based Discriminant Analysisfor Eye Detection", IEEE TRANSACTIONS ON IMAGE PROCESSING, vol. 23, no. 4, (2014) April, pp. 1629-1637.

[5] N. K. Bansode and P. K. Sinha," Facial Feature Extraction and Textual Description Classificationusing SVM",(ICCCI -2014), Coimbatore, (2014) January, pp. 03-05.

[6] I. Paukner Stojkov and S. Loncaric," A method for eye detection in face images", MIPRO, Opatija, Croatia, (2010) May, pp. 24-28

[7] K. Azha, A. Aziz, R. A. Ramlee, S. S. Abdullah and A. N. Jahari, "Face Detection Using Radial Basis Function Neural Networks With VarianceSpread Value", International Conference of Soft Computing and Pattern Recognition, (2010).

[8] R. J. T. Morris, L. D. Rubin and H. Tirri, "Neural network techniques for object orientation detection: solution by optimal feed-forward network and learning vector quantization approaches", IEEE Trans. Pattern Anal. Mach. Intell., vol. 12, no. 11, (1990), pp. 1107-1115. 
International Journal of Signal Processing, Image Processing and Pattern Recognition Vol. 9, No. 6 (2016) 